\title{
Ultrasound Characterization of Three Animal MAMmary Tumors From THREE-Dimensional ACOUSTIC Tissue Models
}

\author{
Jonathan Mamou ${ }^{1}$, Michael L. Oelze ${ }^{2}$, William D. O’Brien Jr. ${ }^{2,3}$, and James F. Zachary ${ }^{3,4}$. \\ ${ }^{1}$ Frederic L. Lizzi Center for Biomedical Engineering, Riverside Research Institute New York, NY. \\ ${ }^{2}$ Department of Electrical and Computer Engineering, ${ }^{3}$ Department of Bioengineering, \\ ${ }^{4}$ Department of Pathobiology, University of Illinois at Urbana-Champaign
}

\begin{abstract}
With very few exceptions, the tissue microstructures responsible for ultrasonic scattering remain unidentified. Identification of these structures would lead to potentially improved methodologies for characterizing tissue and diagnosing disease from ultrasonic backscatter measurements. Three-dimensional (3D) acoustic models of tissue microstructure, termed 3D impedance maps (3DZMs) were obtained for three tumors (a rat fibroadenoma, a mouse carcinoma, and a mouse sarcoma). 3DZMs were inferred from a 3D histological data set of tissue, and are independent of ultrasonically acquired data. The Fourier transform of the 3D spatial autocorrelation of the 3DZM was used to estimate the backscattered power spectrum. Spectral estimates (scatterer size and acoustic concentration) were then obtained by fitting an assumed scattering model (form factor, FF) to the power spectrum. At first, a Gaussian FF was used to allow comparison with the independent in vivo measurements from the same three tumor tissues. Then, a tissue-specific FF was deduced from each 3DZM. The 3DZM estimates using the Gaussian FF led to scatterer estimates very similar (within 10\%) to those obtained with the independent ultrasonic backscatter measurements. However, a clear distinction could not be made between the sarcoma and carcinoma using the estimates obtained with the Gaussian FF. Statistical distinction was finally obtained when the 3DZM-deduced FF was used to obtain estimates. Thus, 3DZMs are unique computational phantoms that can to help identify ultrasonic scattering sites, and to develop advanced models and sensitive ultrasonic techniques to diagnose disease.
\end{abstract}

\section{INTRODUCTION}

The echoes or backscattered signals received by an ultrasonic transducer used to image tissues arise from small spatial variations in the mechanical properties of the tissues. Quantitative ultrasound (QUS) strategies try to improve conventional ultrasonic B-mode images by displaying quantitative information about structures smaller than the wavelength of the incident ultrasound. QUS parameters are estimated from the frequency- dependencies of the acquired backscattered RF-data [1][3]. Two QUS parameters are often estimated from the spectrum of the backscattered signals, the scatterer size and the acoustic concentration (product of the number density and the relative impedance mismatch of the scatterers squared) [4], [5].

The QUS estimates depend on the theoretical model chosen to describe the ultrasonic scattering. The functions that describe the scattering structures are called form factors (FF). Choosing a FF to model breast cancer is not a straightforward task because the anatomic structures responsible for ultrasonic scattering remain unidentified.

A method is proposed to obtain a tissue-specific FF that is directly based on morphological acoustic modeling of tumor tissue. This method involves the development of 3D impedance maps (3DZMs) of tumor tissue. QUS estimates then can be obtained with the tissue-specific FF and compared to those obtained with conventional FFs (e.g., Gaussian [2], [6]). In particular, if successful, a specific FF would lead to improved contrast in the estimates. This contrast could then translate into a significant improvement in diagnostic ultrasound imaging.

\section{Methods}

\section{A. 3D Impedance Maps}

A 3D impedance map can be seen as a computational phantom. It is essentially a 3D matrix that describes the acoustical properties of a 3D medium; the elements are the values of the acoustical impedance of the medium.

Tumors were fixed (10\% neutral-buffered formalin), embedded in paraffin, serially sectioned, and stained with $\mathrm{H} \& \mathrm{E}$ for histologic evaluation. Each section was digitally photographed through the light microscope (Nikon Optophot-2). 3DZMs were then obtained after contrast equalization of each section, registration of adjacent sections, interpolation of the sections lost during 
(a)

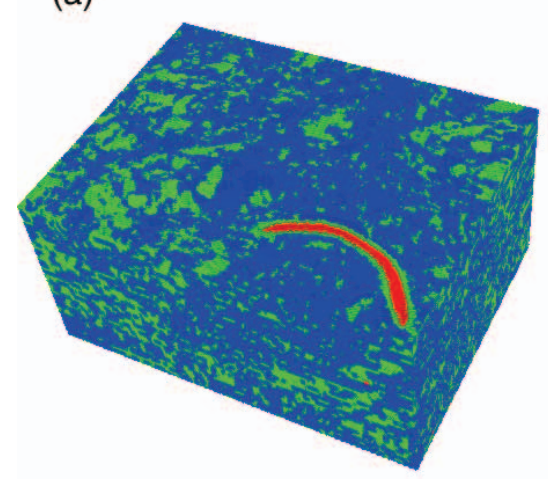

(b)

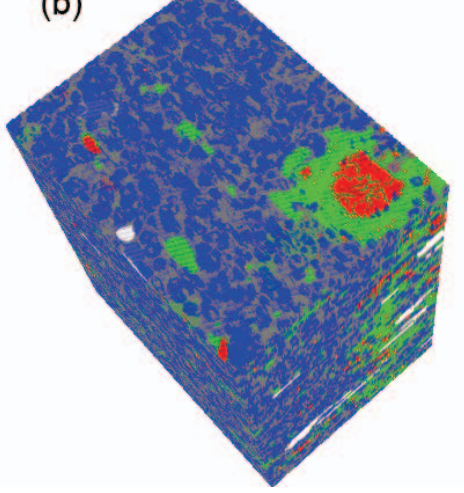

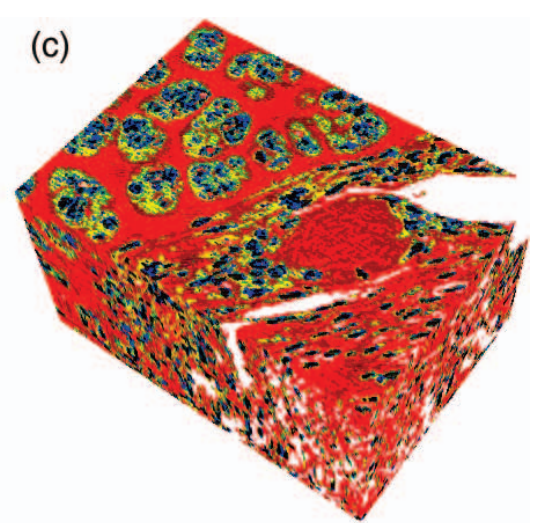

Fig. 1. Three-dimensional impedance maps renderings of the fibroadenoma (a), the carcinoma (b), and the sarcoma (c). Each color represents an identified tissue microstructures with a unique impedance value (Table I).

sectioning, and assignment of acoustic impedance values to recognized tissue microstructures (e.g., cytoplasm, nuclei, fat, etc). Acoustic impedance values were somewhat empirical but inferred from the literature (see Ref. [8]).

In this study, three tumors were investigated, a rat fibroadenoma, a mouse carcinoma, and a mouse sarcoma (see Fig. 1). The 3DZM sizes and impedance values are reported in the first two rows of Table I.

\section{B. Estimation of scatterer size and acoustic concentra- tion}

The backscattered intensity, $I_{b s}$, due to an incident plane wave on a weakly inhomogeneous medium is described by [7]:

$$
\begin{aligned}
I_{b s} & =A k^{4} S(2 k) \text { where } \\
S(2 k) & =\left|\iiint_{V_{0}} \frac{\Delta z(r)}{z(r)} e^{-2 j k r_{0}} d v_{0}\right|^{2} .
\end{aligned}
$$

$A$ is a proportionality constant and $k$ is the wave number. Hence, the power spectrum of the relative impedance variations can be easily extracted from the backscattered intensity.

The 3D impedance map is divided into smaller 3D volumes called regions of interest (ROIs). The power spectrum, $S(2 k)$, of each ROI is computed by taking the magnitude squared of the Fourier transform of the ROI. Then, an optimization scheme is used to fit the chosen FF on $S(2 k)$ [8]. The scatterer size and the acoustic concentration are readily deduced from the fitting parameters. Because the acoustic concentration has generally a large dynamic range, it is transformed into a log scale through $C_{d B}=10 * \log \left(\mathrm{C}\left[\mathrm{mm}^{-3}\right]\right)$.

\section{Form factor extraction}

Let us assume that a given 3DZM contains a single type of scatterer but with variations in size and in local acoustic concentration. Ideally, it is desirable to derive a FF from the available 3DZM that would be an accurate scattering model for this assumed single type of scatterer.

Assuming that the 3DZM is divided into $N$ ROIs and denoting the power spectrum of the $l^{\text {th }}$ ROI by $P S_{l}$. Each $P S_{l}$ will be transformed to $T_{l}\left(P S_{l}\right)$ using a transformation $\left(T_{l}\right)$ with two degrees of freedom (magnitude scaling and frequency-axis scaling) to account for the two unknown parameters (scatterer size and acoustic concentration). A set of $N$ transforms then can be found such that each transformed power spectrum is the same because each ROI contains the same scattering structures. Therefore, a fitting criterion is introduced to quantify with a single number the similarity of the $\mathrm{N}$ transformed power spectra. This criterion is the mean standard deviation (MSTD) of the transformed power spectra over the chosen frequency range. The quantity that needs to be minimized is

$$
\operatorname{MSTD}=\frac{1}{k_{2}-k_{1}} \int_{k_{1}}^{k_{2}} U(k) \mathrm{dk},
$$

where

$$
U(k)=\sqrt{\sum_{l=1}^{N}\left[T_{l}\left(P S_{l}\right)(k)-\langle T(P S)(k)\rangle\right]^{2}} .
$$

$\langle T(P S)(k)\rangle$ is the mean of the transformed power spectra and is defined by

$$
\langle T(P S)(k)\rangle=\frac{1}{N} \sum_{l=1}^{N} T_{l}\left(P S_{l}\right)(k) .
$$


TABLE I

SUMMARY OF THE ESTIMATES OBTAINED FROM THREE TUMORS.

\begin{tabular}{|c|c|c|c|}
\hline & Rat fibraodenoma & Mouse carcinoma & Mouse sarcoma \\
\hline \multirow{3}{*}{$\begin{array}{c}\text { Impedance values } \\
\text { (Mrayl) }\end{array}$} & 1.58 - epithelial cells (green) & 1.45 - fat (white) & 2.00 - nuclear heterocrhomatin (black) \\
\hline & 1.80 - connect. tissue (blue) & 1.60 - nuclei (blue) & $1.70 / 1.65$ - extracell. matrix (dark red/red) \\
\hline & & 1.80 - connective tissue (green) & $1.58 / 1.55$ - cytoplasm (green/yellow) \\
\hline 3DZM size $(\mu \mathrm{m})$ & $800 * 600 * 390$ & $200 * 150 * 330$ & $218 * 156 * 129$ \\
\hline Ultrasound estimates & $<\mathrm{D}>=105 \pm 25 \mu \mathrm{m}$ & $<\mathrm{D}>=30.0 \pm 9.6 \mu \mathrm{m}$ & $\langle\mathrm{D}\rangle=33 \pm 8.0 \mu \mathrm{m}$ \\
\hline Gaussian FF & $<n z^{2}>=-21.9 \pm 6.1 \mathrm{~dB}\left(\mathrm{~mm}^{-3}\right)$ & $<n z^{2}>=-1.4 \pm 6.1 \mathrm{~dB}\left(\mathrm{~mm}^{-3}\right)$ & $<n z^{2}>=-0.59 \pm 4.3 \mathrm{~dB}\left(\mathrm{~mm}^{-3}\right)$ \\
\hline 3DZM estimates & $<\mathrm{D}\rangle=96.5 \pm 14 \mu \mathrm{m}$ & $\langle\mathrm{D}\rangle=56.0 \pm 19 \mu \mathrm{m}$ & $<\mathrm{D}>=37.0 \pm 12 \mu \mathrm{m}$ \\
\hline Deduced FF & $<n z^{2}>=-21.8 \pm 5.1 \mathrm{~dB}\left(\mathrm{~mm}^{-3}\right)$ & $<n z^{2}>=-6.9 \pm 7.2 \mathrm{~dB}\left(\mathrm{~mm}^{-3}\right)$ & $<n z^{2}>=0.46 \pm 5.5 \mathrm{~dB}\left(\mathrm{~mm}^{-3}\right)$ \\
\hline
\end{tabular}

The algorithm finishes when the $\mathrm{N}$ transforms (i.e., $T_{1},-$ ,$T_{N}$ ) that minimize the MSTD (Eq. (3)) have been found. The normalized mean power spectrum $\frac{\langle T(P S)(k)\rangle}{\langle T(P S)(0)\rangle}$ is taken as the estimated tissue-specific FF.

\section{RESULTS}

\section{A. Simulations}

Two simulations were conducted to evaluate the FF extraction algorithm. The first simulation consisted of 25 ROIs containing a random number of randomly located scatterers. Each scatterer followed a Gaussian impedance distribution and the scatterers within all the ROIs had the same diameter of $32 \mu \mathrm{m}$. This simulation was meant to test the methodology because in this case, the optimal FF is the Gaussian FF. Figure 2 shows the actual sizes (diameters), the Gaussian size estimates, and the size estimates obtained with deduced FF for each of the 25 ROIs. This figure shows that the accuracy of the estimates is excellent; all the size estimates (Gaussian FF and deduced FF) are in the range 29-35 $\mu \mathrm{m}$ (i.e., within $10 \%$ ). There is no visible difference in accuracy between the Gaussian estimates and the deduced FF estimates. Some Gaussian estimates are more accurate and some less accurate than the deduced FF estimates. The mean errors were $0.81 \mu \mathrm{m}$ and $0.77 \mu \mathrm{m}$ for the Gaussian and deduced FFs, respectively. These results tend to verify that for simple simulation, the FF-extraction algorithm was capable of deriving an accurate FF.

The second simulated 3DZM was also divided into 25 ROIs. Each ROI contained a normal distribution of sizes where the mean radius was random but the standard deviation was $75 \%$ of the mean radius. Radii were randomly selected in the large range 8-24 $\mu \mathrm{m}$ (i.e., diameter randomly selected in the range 16-48 $\mu \mathrm{m}$ ). Each scatterer followed a Gaussian impedance distribution.

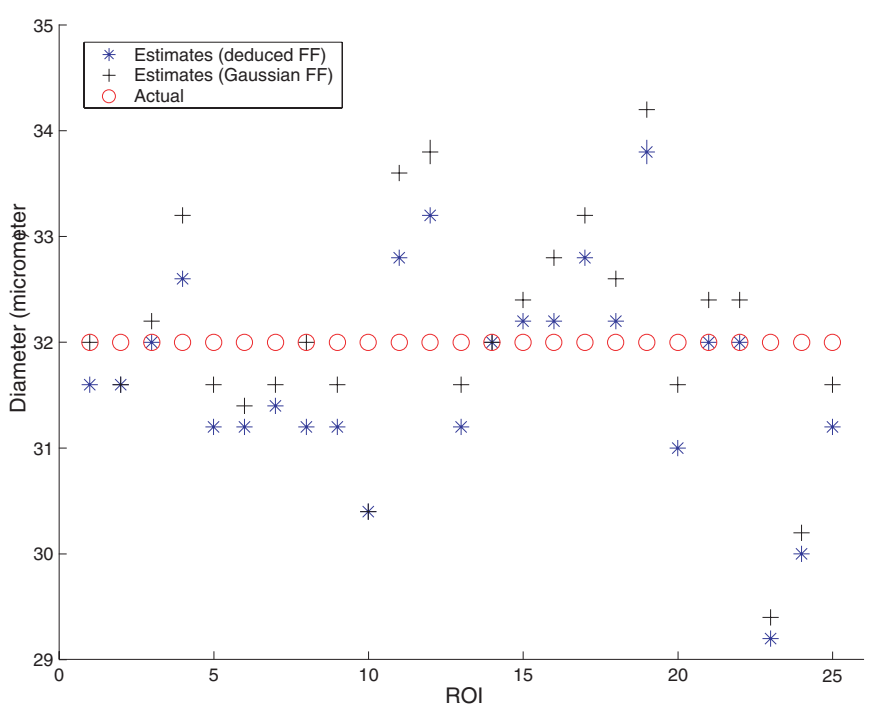

Fig. 2. Estimates obtained from 25 ROIs using the Gaussian and the 3DZM-deduced FF. Each ROI was filled with a random number of randomly located Gaussian scatterers. Each ROI contained scatterers of diameter $32 \mu \mathrm{m}$.

For this simulation, the Gaussian FF was expected to perform poorly and to greatly overestimate scatterer diameters because of the standard deviation effect [8]. The Gaussian estimates always were greater than the actual values, i.e., the Gaussian estimates are biased (Figure 3). The mean Gaussian estimate bias (same as error for this case) was $+9.2 \mu \mathrm{m}(+29 \%)$. In contrast, the deduced FF estimates had no appreciable bias (some estimates were greater and some were less than the actual value) and were accurate. The mean error for the deduced FF estimates was $2.0 \mu \mathrm{m}$. 


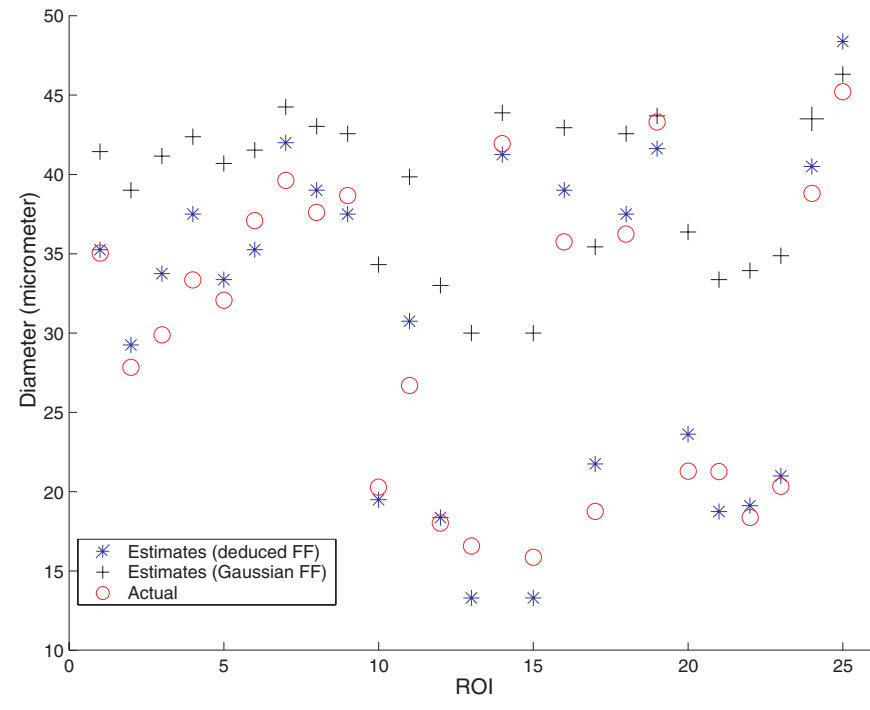

Fig. 3. Estimates obtained from 25 ROIs using the Gaussian and the 3DZM-deduced FF. Each ROI contained scatterers following a Gaussian size distribution. For each ROI, the scatterers mean diameter was chosen randomly in the range 16-48 $\mu \mathrm{m}$ and the standard deviation was $75 \%$ of the mean diameter.

\section{B. Tumor models}

The FF-extraction strategy was then conducted for the three tumor 3DZMs. Size and acoustic concentration estimates were obtained using the Gaussian FF and the deduced FF. Estimates are displayed alongside with those obtained in vivo using a Gaussian FF (the last three rows of Table I).

The results showed good agreement between the two independent techniques for scatterer size estimates using a Gaussian FF. However, significant differences were observed for the acoustic concentration values. Furthermore, using a Gaussian FF, neither the 3DZM nor the in vivo estimates exhibited a statistical difference between the sarcoma and the carcinoma. However, a clear distinction was found when the deduced FF was used.

\section{Conclusions}

A method was developed to obtain tissue-specific models (i.e., FF) of ultrasonic scattering. This method involves the development of 3D impedance maps from histologic sections of tumor tissues.

The FF-extraction methodology was validated on two simulated 3DZMs. The results showed that the algorithm was capable of accurately extracting a FF because the errors in the size estimates using the extracted FF were small $(<7 \%)$. In particular, for the second simulation, the use of the Gaussian FF had poor accuracy and the sizes were greatly overestimated (by 29\%).

The FF-extraction strategy was then used with three 3D impedance maps derived from three different tumors, a rat fibroadenoma, a mouse carcinoma, and a mouse sarcoma. The scatterer-size estimates using the extracted FF allowed for a statistical distinction among the three tumors. However, discriminating between the carcinoma and the sarcoma was not possible based on the estimates obtained using a Gaussian FF.

The results demonstrate that the 3DZMs can be a valuable tool in developing tissue-specific scattering models. These specific models will improve the diagnosis potential of QUS methodologies.

\section{ACKNOWLEDGEMENT}

Support provided by the University of Illinois Research Board.

\section{REFERENCES}

[1] M. F. Insana, R. F. Wagner, and D. G. Brown, "Describing smallscale structure in random media using pulse-echo ultrasound," $J$. Acoust. Soc. Am., vol. 87, pp. 179-192, 1990.

[2] F. L. Lizzi, M. Greenebaum, E. J. Feleppa, M. Elbaum, and D. J. Coleman, "Theoretical framework for spectrum analysis in ultrasonic tissue characterization," J. Acoust. Soc. Am., vol. 73, pp. 1366-1373, April 1983.

[3] E. J. Feleppa, F. L. Lizzi, D. J. Coleman, and M. M. Yaremko, "Diagnostics spectrum analysis in ophthalmology: A physical perspective," Ultrasound Med. Biol., vol. 12, pp. 623-631, 1986.

[4] F. L. Lizzi, M. Ostromogilsky, E. J. Felepa, M. C. Rorke, and M. M. Yaremko, "Relationship of ultrasonic spectral parameters to features of tissue microstructure," IEEE Trans. Ulatrson. Ferroelectr. Freq. Control, vol. 33, pp. 319-329, May 1986.

[5] M. L. Oelze, J. F. Zachary, and W. D. O'Brien, Jr., "Characterization of tissue microstructure using ultrasonic backscatter: Theory and technique for optimization using a Gaussian form factor," J. Acoust. Soc. Am., vol. 112, pp. 1202-1211, 2002.

[6] M. L. Oelze, J. F. Zachary, and W. D. O'Brien, Jr., "Parametric imaging of rat mammary tumors in vivo for the purposes of tissue characterization," J. Ultrasound Med., vol. 21, pp. 1201-1210, 2002.

[7] P. M. Morse and K. U. Ingard, Theoretical Acoustics, McGrawHill, New York, 1968.

[8] J. Mamou, M. L. Oelze, W. D. O'Brien, Jr., and J. F. Zachary, "Identifying ultrasonic scattering sites from three-dimensional impedance maps," J. Acoust. Soc. Am., vol. 117, pp. 413-423, 2005. 